\title{
STONE OPERATOR MINING FACTS - 2007
}

\section{Mining Operations}

In 2007, a total of 4,639 stone mining operations reported employment to the Mine Safety and Health Administration (MSHA). ${ }^{1}$ Stone mines comprised $31.2 \%$ of all mining operations.

- Mines producing the primary commodity of limestone (crushed and broken) comprised the largest number of operations $(n=2,051 ; 44.2 \%)$.

- Stone mining operations were located in all states and territories except Delaware and North Dakota.

- Pennsylvania had the most stone mines $(n=373$; $8.0 \%)$, followed by Texas $(n=352 ; 7.6 \%)$.

\section{Employees}

A total of 82,718 employees, ${ }^{2}$ corresponding to 83,254 full-time equivalent (FTE) ${ }^{3}$ employees, were reported to MSHA by stone mine operators in 2007.

- Within the mining sectors, ${ }^{4}$ stone mine operator employees accounted for $24.7 \%$ of all employee hours reported.

- Stone operator employee hours were reported for both underground (2.5\%) and surface (97.5\%) work locations. ${ }^{5}$

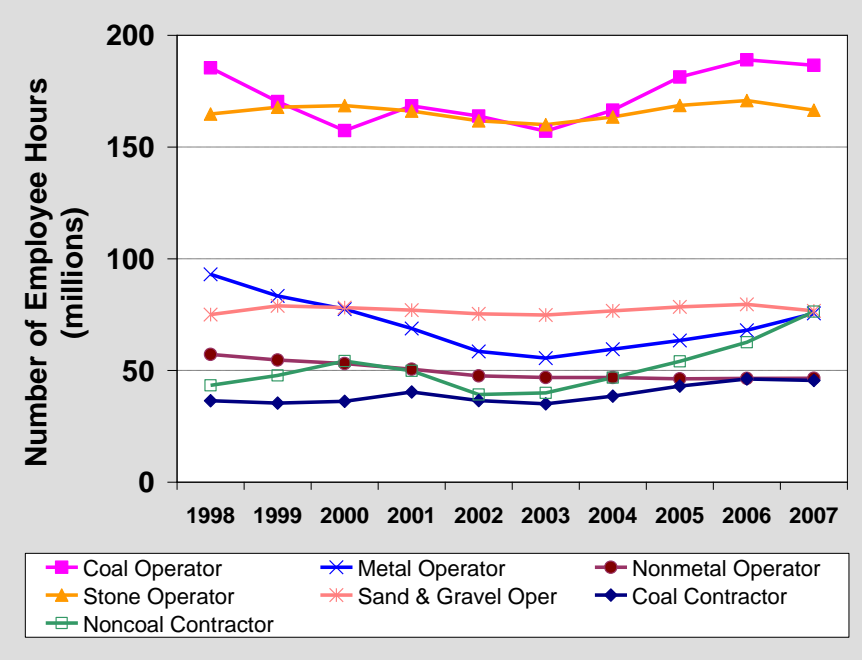

\section{Fatalities}

There were eight work-related fatalities among stone mine operator employees in 2007. These fatalities all occurred at surface work locations.

- The stone mine operator fatality rate for surface work locations was 11.4 fatalities per 100,000 FTE employees.

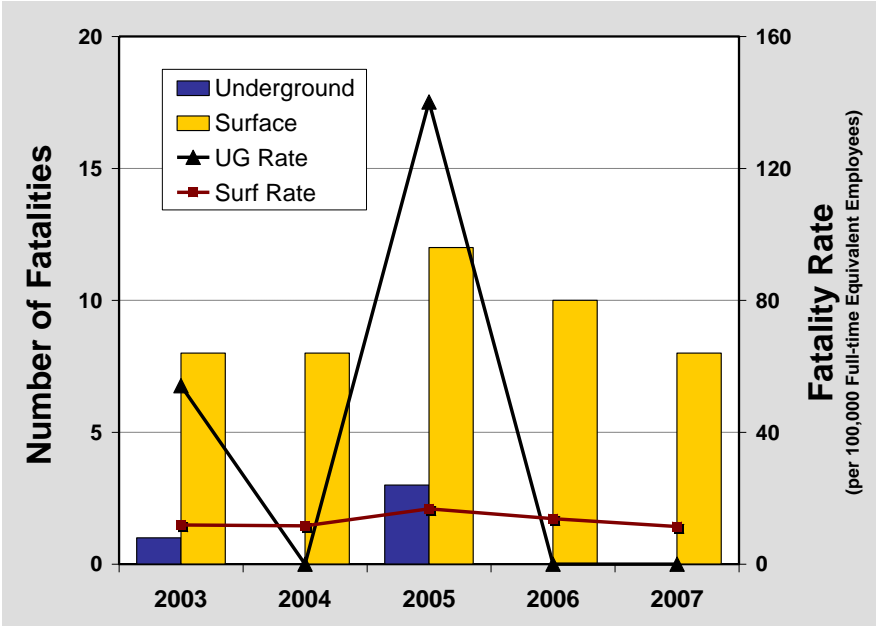

Nonfatal Lost-time Injuries

There were 1,880 nonfatal lost-time injuries (36 at underground and 1,844 at surface work locations) among stone operator employees occurring at an overall rate of 2.6 injuries per 100 FTE employees. A total of 85,639 days lost from work ${ }^{6}$ resulted from these injuries, comprising $20.1 \%$ of days lost across all mining sectors.

- The nonfatal lost-time injury rate was 1.7 for underground and 2.6 for surface work locations.

- The most frequent classification of nonfatal losttime injuries for stone operator employees involved handling materials $(n=689 ; 36.6 \%)$.

- Sprains and strains were the most frequently reported nature of injury $(n=843 ; 44.8 \%)$.

- The back was the most frequently reported body part injured $(n=378 ; 20.1 \%)$ and accounted for 15,317 days lost from work.

\section{Distribution of Nonfatal Lost-time Injuries by Accident Class, $2007(\mathrm{~N}=1,880)$}

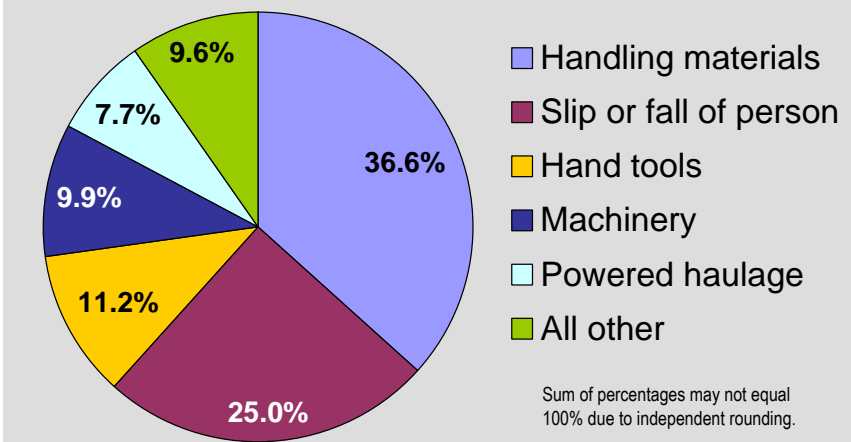

Note: "Fall of ground" includes MSHA's Accident/lnjury/lllness class for fall of face, rib, pillar, side, or highwall; fall of roof, back, or brow; and machinery cases when the source of injury was caving rock, coal, ore, or waste. 


\section{Mine and Employment Characteristics, 2007}

\begin{tabular}{|c|c|c|c|c|c|c|c|c|c|}
\hline \multirow{2}{*}{$\begin{array}{l}\text { Commodity and } \\
\text { Type of Employer }\end{array}$} & \multicolumn{3}{|c|}{ Number of Mining Operations ${ }^{1}$} & \multicolumn{3}{|c|}{ Number of Employees ${ }^{2}$} & \multicolumn{3}{|c|}{ Number of FTE Employees ${ }^{3}$} \\
\hline & Underground & Surface & Total & Underground & Surface & Total & Underground & Surface & Total \\
\hline Coal Operator & 631 & 1,399 & 2,030 & 37,829 & 46,242 & 84,071 & 42,238 & 51,027 & 93,265 \\
\hline Metal Operator & 97 & 181 & 278 & 5,333 & 30,667 & 36,000 & 5,437 & 32,309 & 37,746 \\
\hline Nonmetal Operator & 48 & 677 & 725 & 2,411 & 20,453 & 22,864 & 2,478 & 20,788 & 23,265 \\
\hline Stone Operator & 113 & 4,526 & 4,639 & 1,845 & 80,873 & 82,718 & 2,063 & 81,191 & 83,254 \\
\hline Sand and Gravel Operator & NA & 7,199 & 7,199 & NA & 45,761 & 45,761 & NA & 38,340 & 38,340 \\
\hline \multirow[t]{2}{*}{ Operator Total } & 889 & 13,982 & 14,871 & 47,418 & 223,996 & 271,414 & 52,216 & 223,655 & 275,871 \\
\hline & \multicolumn{3}{|c|}{ Number of Companies } & & & & & & \\
\hline Coal Contractor & NA & NA & 2,999 & 5,160 & 33,705 & 38,865 & 3,071 & 19,670 & 22,741 \\
\hline Noncoal Contractor & NA & NA & 5,400 & 3,039 & 64,805 & 67,844 & 1,935 & 36,216 & 38,151 \\
\hline Contractor Total & NA & NA & 8,399 & 8,199 & 98,510 & 106,709 & 5,006 & 55,886 & 60,892 \\
\hline \multicolumn{4}{|l|}{ TOTAL } & 55,617 & 322,506 & 378,123 & 57,222 & 279,541 & 336,763 \\
\hline
\end{tabular}

Mining Occupational Fatalities and Injuries, 2007

\begin{tabular}{|c|c|c|c|c|c|c|c|c|c|c|c|c|}
\hline \multirow{3}{*}{$\begin{array}{l}\text { Commodity and } \\
\text { Type of Employer }\end{array}$} & \multicolumn{6}{|c|}{ Occupational Fatalities (per 100,000 FTE employees) } & \multicolumn{6}{|c|}{ Nonfatal Lost-time Injuries (per 100 FTE employees) } \\
\hline & \multicolumn{2}{|c|}{ Underground } & \multicolumn{2}{|c|}{ Surface } & \multicolumn{2}{|c|}{ Total } & \multicolumn{2}{|c|}{ Underground } & \multicolumn{2}{|c|}{ Surface } & \multicolumn{2}{|c|}{ Total } \\
\hline & No. & Rate & No. & Rate & No. & Rate & No. & Rate & No. & Rate & No. & Rate \\
\hline Coal Operator & 18 & 42.6 & 10 & 21.1 & 28 & 31.2 & 2,120 & 5.0 & 822 & 1.7 & 2,942 & 3.3 \\
\hline Metal Operator & 3 & 55.2 & 4 & 14.4 & 7 & 21.1 & 178 & 3.3 & 620 & 2.2 & 798 & 2.4 \\
\hline Nonmetal Operator & 1 & 40.4 & 0 & 0.0 & 1 & 5.0 & 91 & 3.7 & 417 & 2.4 & 508 & 2.5 \\
\hline Stone Operator & 0 & 0.0 & 8 & 11.4 & 8 & 11.1 & 36 & 1.7 & 1,844 & 2.6 & 1,880 & 2.6 \\
\hline Sand and Gravel Operator & NA & NA & 5 & 15.0 & 5 & 15.0 & NA & NA & 657 & 2.0 & 657 & 2.0 \\
\hline Operator Total & 22 & 42.1 & 27 & 13.8 & 49 & 19.7 & 2,425 & 4.6 & 4,360 & 2.2 & 6,785 & 2.7 \\
\hline Coal Contractor & 3 & 97.7 & 3 & 16.3 & 6 & 28.0 & 177 & 5.8 & 283 & 1.5 & 460 & 2.1 \\
\hline Noncoal Contractor & 2 & 103.4 & 10 & 29.3 & 12 & 33.3 & 43 & 2.2 & 454 & 1.3 & 497 & 1.4 \\
\hline Contractor Total & 5 & 99.9 & 13 & 24.8 & 18 & 31.3 & 220 & 4.4 & 737 & 1.4 & 957 & 1.7 \\
\hline TOTAL & 27 & 47.2 & 40 & 16.1 & 67 & 21.9 & 2,645 & 4.6 & 5,097 & 2.0 & 7,742 & 2.5 \\
\hline
\end{tabular}

NA Not applicable.

Data source: Publicly released data files of employment and accident/injury/illness collected by MSHA under 30 CFR 50 .

Notes: All analyses of accident data exclude office employees. Occupational fatalities exclude all cases under 17 years of age. Further statistical methodology is available on the NIOSH Internet [http://www.cdc.gov/niosh/mining/statistics/method.htm]. Data in the above tables may not add to totals shown because of independent rounding. Caution should be used when interpreting rates based on a small number of events.

${ }^{1}$ Mines at which only independent contractors were working did not show any employment and were not counted.

${ }^{2}$ Average number of employees working at individual mines during calendar quarters of active operations (includes office workers).

${ }^{3}$ Full-time equivalent employees computed using reported employee hours $(2,000$ hours $=1$ FTE $)$.

${ }^{4}$ Mining sectors include coal operators, metal operators, nonmetal operators, stone operators, sand and gravel operators, coal contractors, and noncoal contractors.

${ }^{5}$ Surface work locations include surface operations at underground mines, surface operations (strip or open pit), dredge, other surface operations, independent shops and yards, and mills or preparation plants.

${ }^{6}$ Includes actual days away from work and/or days of restricted work activity. For permanently disabling injuries only, statutory days charged by MSHA were used if they exceeded the total lost workdays.

To receive $\mathrm{NIOSH}$ documents or more information about occupational safety and health topics, contact $\mathrm{NIOSH}$ at

1-800-CDC-INFO (1-800-232-4636)

TTY: 1-888-232-6348

e-mail: cdcinfo@cdc.gov

or visit the NIOSH Web site at http://www.cdc.gov/niosh 